\title{
Solid phase synthesis of novel pyrazole derivatives from diaryl 1,3-diketones under microwave irradiation
}

\author{
Suresh Singh Chauhan, A. Sharma, S. Saingar and Y. C. Joshi \\ Department of Chemistry, University of Rajasthan, Jaipur-302 004, India \\ E-mail : sureshsingh76@yahoo.com
}

Manuscript received I February 2005, revised 8 June 2005, accepted 18 July 2005

Microwave irradiation is increasingly being used to accelerate the rate of reaction'. In this paper we wish to report, reaction of diaryl-1,3-diketone ${ }^{2}$ (1a-1) with hydrazine on silica gel under microwave irradiation to generate novel pyrazole derivatives.

Microwave irradiation of propane-1-(1,3-benzodioxol5-yl)-3-pheny-1,3-dione (1a-l) with hydrazine hydrate on silica gel gives Schiff bases type compound having novel pyrazole nucleus. Hydrazine hydrate promote the enol form of mentioned diketone. The structure of the solid phase synthesized compound are well supported by spectroscopic data (Scheme 1, Table 1).

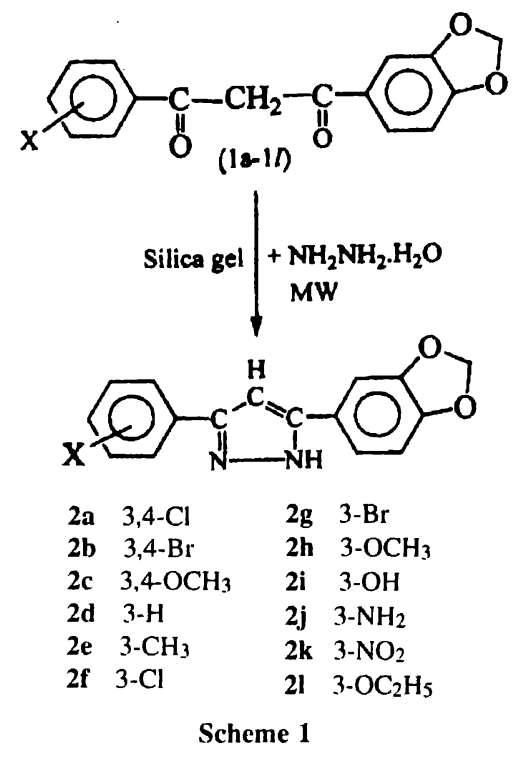

\section{Results and discussion}

In IR spectra, characteristic -NH stretching vibration was observed around $3250 \mathrm{~cm}^{-1}$, $-\mathrm{CN}$ stretching band around $1420 \mathrm{~cm}^{-1}$. symmetric and asymmetric vibration bands appearing in the region 1020-1075 and 1265-1235 $\mathrm{cm}^{-1}$ respectively. In ${ }^{1} \mathrm{H}$ NMR all the solid phase synthesized compounds exhibit a single sharp peak in the region $\delta$ 1016
$7.21-7.34$ due $-\mathrm{NH}$ proton. A singlet at $\delta 5.98-6.01$ is due to dioxymethylene group. Multiplets at $\delta 6.6-8.3$ indicates the presence aromatic protons. A singlet at $\delta 3.79$ is observed due to the $-\mathrm{OCH}_{3}$ group protons. 'H NMR data of the title compound and ${ }^{13} \mathrm{C}$ NMR data are presented in Table 1.

In the mass spectra of these title compounds (2a-1) shows molecular ion peaks $(\mathrm{m} / \mathrm{z})$. The $[\mathrm{M}+2]^{+}$peaks was nearly one fourth of $[\mathrm{M}]^{+}$peaks indicating the presence isotopic $\mathrm{Cl}^{37}$, and the compound (2d) shows a cluster of peaks at $m / z 343$ and 345 corresponding to $\mathrm{M}^{+}$and $[\mathrm{M}+2]^{+}$respectively. $\mathrm{M}^{+}$and $[\mathrm{M}+2]^{+}$peaks have same intensity showing the presence of bromine. A culture of ion peaks to $[\mathrm{M}]^{+}$, $[\mathrm{M}+2]^{+}$at 298,300 were observed in case $(2 \mathrm{c})$.

The ${ }^{13} \mathrm{C}$ NMR data for the compound (2a-l) are presented in Table 1 , and these data are in good agreement with their structure.

\section{Experimental}

Melting points are uncorrected. The IR spectra were recorded in $\mathrm{KBr}$ disk on a Nicolet Megna-FT-IR 550 spectrometer. ${ }^{1} \mathrm{H}$ NMR and ${ }^{13} \mathrm{C}$ NMR were recorded on model DRX 300 at 300.13 and $75.48 \mathrm{MHz}$, respectively, in $\mathrm{CDCl}_{3} / \mathrm{DMSO}-d_{6}$ using TMS as internal standard. Mass spectra were recorded on Jeol D-300 spectrometer. The purity of the newly synthesized compounds were checked by TLC.

\section{Generalized preparation of pyrazole derivatives:}

Hydrazine hydrate ( $130 \mathrm{mg}, 4 \mathrm{mmol})$, silica gel $(2 \mathrm{~g})$ and diaryl-1,3-diketone $1 \mathrm{a}-\mathrm{l}(2 \mathrm{mmol})$ were mixed in a mortar then transferred in to a beaker and irradiated for $2 \mathrm{~min}$. The progress of reaction were monitored by $\mathrm{TLC}\left(\mathrm{CHCl}_{3}\right.$ : $\left.\mathrm{CH}_{3} \mathrm{OH}, 9: 1\right)$ as mobile phase. The mixture was extracted into $\mathrm{CHCl}_{3}$ then filtered and washed with water, after drying, the organic phase was removed by a rotary evaporator. 
Note

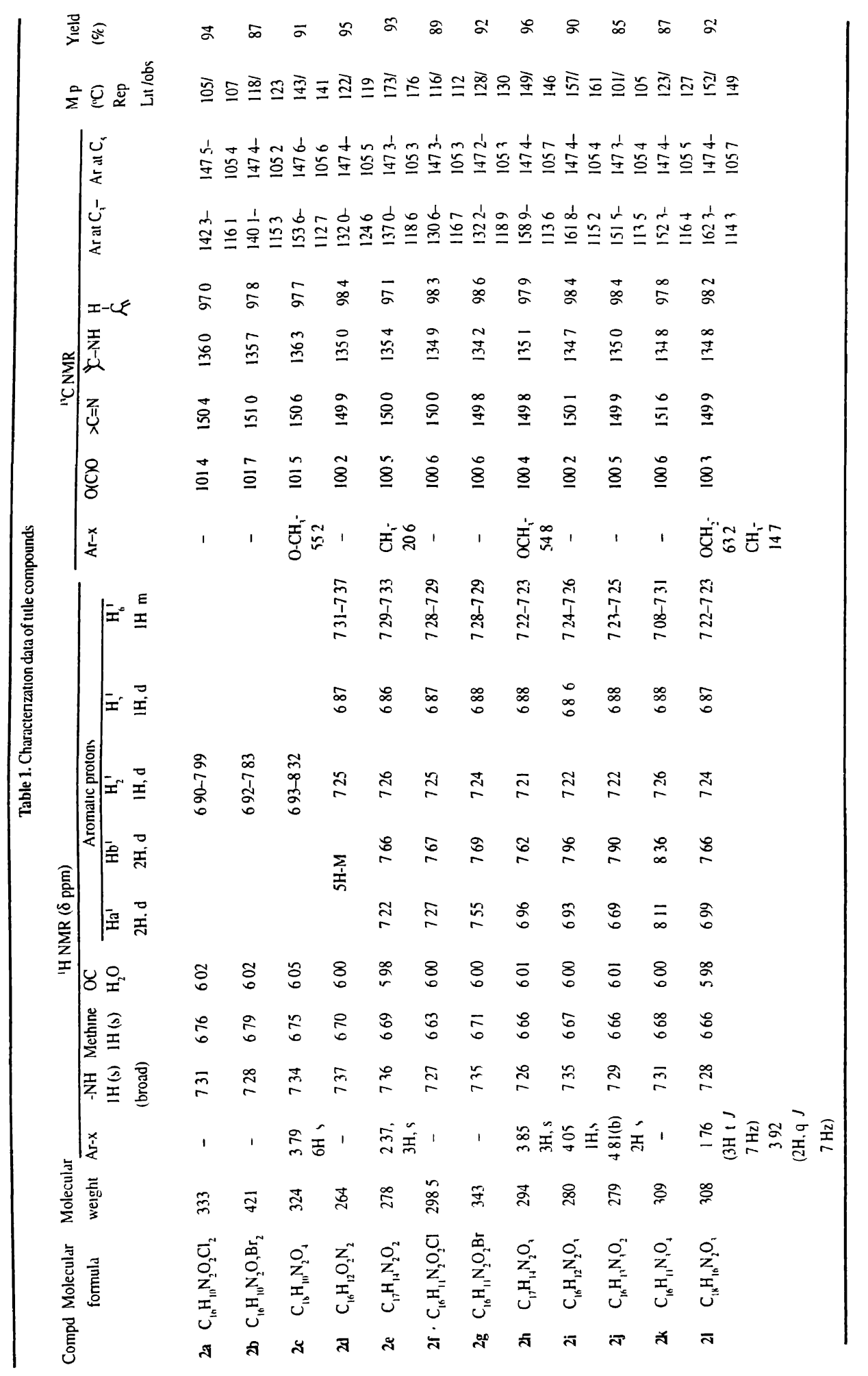


J. Indian Chem. Soc., Vol. 82, November 2005

Further purification by column chromatography $\left(\mathrm{CHCl}_{3}\right.$ facilities. Authors are also thankful to Central Drug Reas eluent) and recrystallization gave the desired products. search Institute, Lucknow for providing spectral data.

\section{Acknowledgement}

Authors are thankful to Head, Department of Chemistry, University of Rajasthan, Jaipur for providing laboratory

\section{References}

1. R. E. Austin et al., Tetrahedron Lett., 2002, 43, 6169.

2. J. Bhagwan, Y. C. Joshi, R. P. Tyagi, B. C. Joshi and H. N. Mangal, J. Institution of Chemist, 1983, 55, 58. 